\title{
Epidural Fluid Collection Originating from Avascular Osteonecrosis Associated with Osteoporotic Vertebral Compression Fracture
}

\author{
Dong Yeob Lee ${ }^{1}$, Hoon Jae Chung ${ }^{2}$ \\ Departments of ${ }^{1}$ Neurosurgery, ${ }^{2}$ Orthopedic Surgery, Bumin Hospital Seoul, Seoul, Republic of Korea
}

Corresponding author: Dong Yeob Lee Department of Neurosurgery, Bumin Hospital Seoul, 389, Gonghang-daero, Gangseo-gu, Seoul 07590, Republic of Korea

Tel: $+82-2-2620-0004$

Fax: +82-2-2620-0167

E-mail: nsdrlee@naver.com

Received: February 5, 2018

Revised: April 2, 2018

Accepted: April 8, 2018
An 83-year-old female complained of intractable right buttock pain and anterior thigh pain after a fall. Magnetic resonance (MR) images revealed an acute osteoporotic compression fracture of the L1 vertebral body with the intravertebral cleft filled with fluid. MR image and MR myelography demonstrated epidural fluid collection causing compression of the cauda equina at the level of the L1 vertebral body, which originated from the intravertebral fluid at the L1 vertebral body. She underwent hemilaminectomy and drainage of epidural fluid, followed by percutaneous vertebroplasty at the L1 vertebral body. Her pain was relieved immediately after the operation. Epidural fluid collection which originated from avascular osteonecrosis associated with an osteoporotic vertebral compression fracture could be a cause of intractable pain in the buttock and the leg, as observed in the present case.

Key Words: Cysts; Epidural space; Fractures; Compression; Lumbar vertebrae; Osteonecrosis

\section{INTRODUCTION}

Osteoporotic vertebral compression fracture (VCF) is a common cause of spine-related disability in the elderly population. Unhealed osteoporotic VCF results in intravertebral avascular osteonecrosis (Kummell's osteonecrosis), which presents as an intravertebral cleft filled with air or fluid ${ }^{8)}$. Avascular osteonecrosis causes spinal instability with progressive collapse of the vertebral body, which commonly presents with intractable back pain requiring surgical intervention ${ }^{5,8,12,13)}$. The optimal surgical treatment for avascular osteonecrosis is still controversial. Some studies have suggested that percutaneous vertebroplasty is enough, but other studies emphasized the need for spinal fusion $^{3,5,6,12,13)}$.

We report a case of epidural fluid collection causing compression of the cauda equina, which originated from avascular osteonecrosis associated with an osteoporotic VCF. Fluid accumulated in the intravertebral cleft leaked into the ventral epidural space, compressed the cauda equina, and finally caused intractable pain in the buttock and the anterior thigh.

\section{CASE REPORT}

An 83-year-old woman developed sudden back pain (Numeric Rating Scale [NRS], 5 points) after a fall. Her back pain was aggravated while turning over and it was markedly relieved in the supine position. She also complained of severe pain in the right buttock and the anterior thigh (NRS, 10 points), which was persistent regardless of her position and did not respond to narcotic analgesics. She could not stand or sit due to severe pain in the right buttock and the anterior thigh. In her past medical history, she was not treated with corticosteroids or for alcohol abuse. Physical examinations revealed absence of motor weakness. The straight leg raising test was negative. Laboratory examinations did not reveal any abnormal findings. $\mathrm{T}$ score for the bone mineral density was -3.8 . A plain lateral radiograph taken in the supine position demonstrated VCF of the L1 and L2 vertebral bodies. Magnetic resonance (MR) images demonstrated an acute osteoporotic VCF with avascular osteonecrosis of the L1 vertebral body. On T2-weighted sagittal and axial images, the content in the intravertebral cleft showed hyperintense signal, suggesting the presence of intravertebral fluid (Fig. 1A, B). There was a ventral epidural cystic lesion compressing the cauda equina at the level of the right side of the L1 vertebral body. The cystic lesion was hyperintense, well-demarcated, ovoid, and $1.8 \mathrm{~cm}$ in size. MR myelography showed that the epidural cystic lesion had a direct connection with fluid accumulated in the intravertebral cleft through a fracture gap in the posterior L1 vertebral body (Fig. 1C). Epidural fluid collection which originated from intravertebral fluid associated with avascular osteonecrosis was diagnosed as the cause of her intractable pain. 


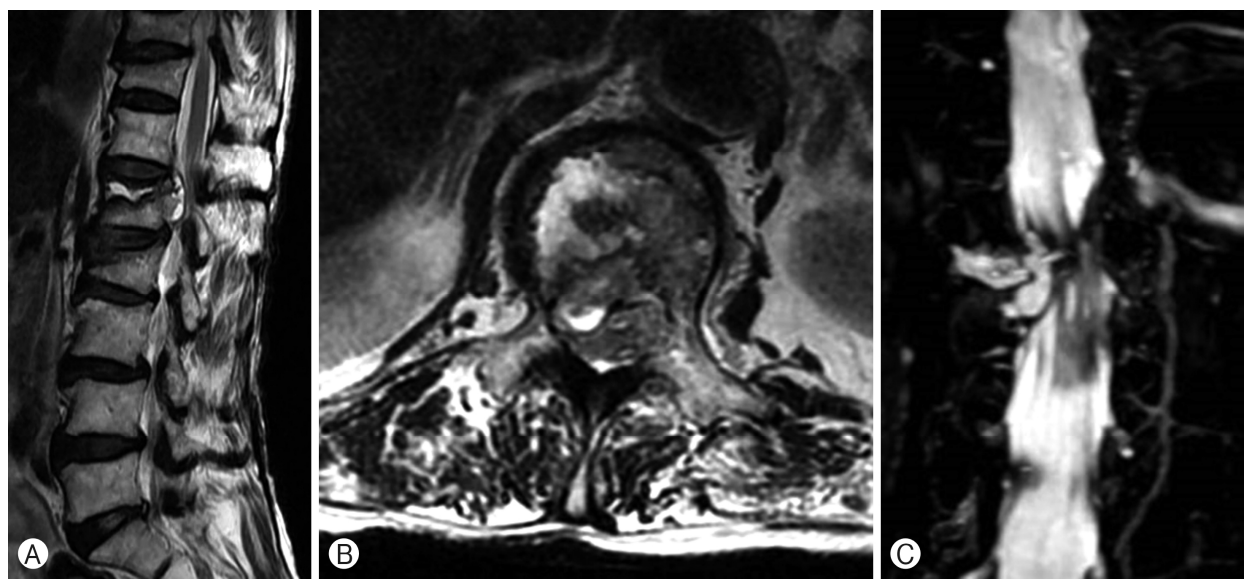

Fig. 1. On T2-weighted sagittal (A) and axial (B) images, the content in the intravertebral cleft showed hyperintense signal, suggesting the presence of the intravertebral fluid. There was a ventral epidural cystic lesion compressing the cauda equina at the level of the right side of the L1 vertebral body. Magnetic resonance myelography $(\mathrm{C})$ clearly showed that the epidural cystic lesion had a direct connection with the fluid in the intravertebral cleft through a fracture gap in the posterior L1 vertebral body.
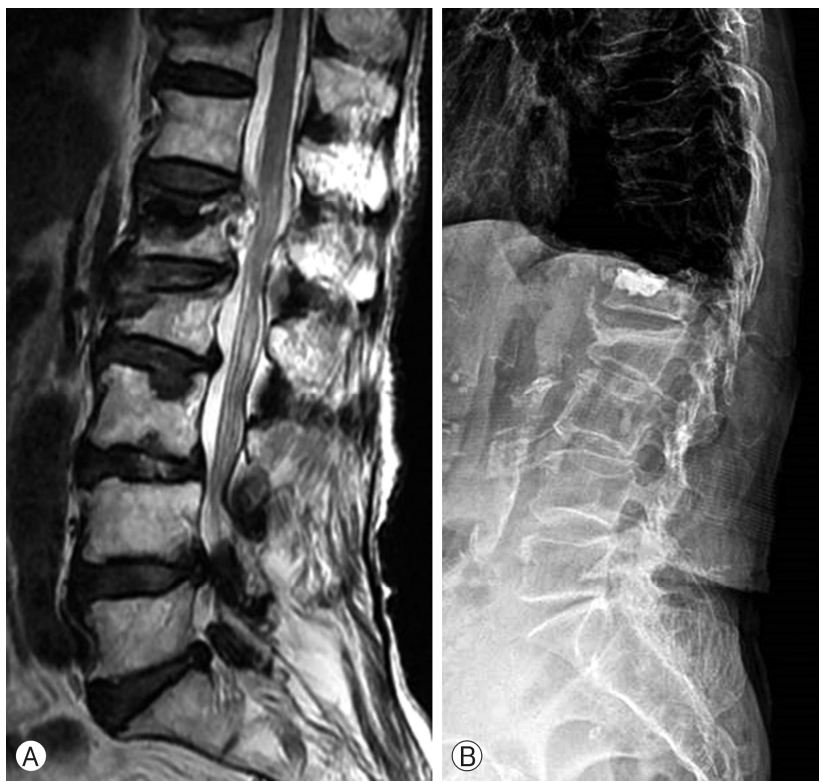

Fig. 2. Magnetic resonance imaging performed on the next day after the operation showed removal of the epidural fluid collection with a well-decompressed cauda equina (A). Plain radiography performed 6 months after the operation showed well-deposited polymethyl methacrylate without recollapse of the augmented vertebral body (B).

The patient underwent operation under general anesthesia in the prone position. After a midline vertical skin incision, the paraspinal muscles were retracted. Hemilaminectomy of the right L1 lamina was performed with a high-speed drill and Kerrison punches. After removal of the ligamentum flavum, the dural sac was carefully exposed. On gentle retraction of the dural sac, clear epidural fluid suddenly gushed out from the ventral side of the dural sac. There was no cystic membrane or capsule-like structure. After complete removal of the epidural fluid collection, the dural sac was fully decompressed. Then, percutaneous vertebroplasty was performed on the right side of the L1 vertebral body. Three cc of polymethyl methacrylate (PMMA) was injected into the intravertebral cleft under C-arm guidance. There was no leakage of PMMA into the epidural space. After insertion of a drain, closure was performed.

Immediately after the operation, her pain markedly improved. MR image performed on the next day after the operation showed removal of the epidural fluid collection with a welldecompressed cauda equina (Fig. 2A). Fluid that had accumulated in the intravertebral cleft nearly disappeared, and PMMA had filled the intravertebral cleft. Two days after the operation, she could stand and walk. Six months after the operation, she only experienced mild pain in her back (NRS, 2 points) and tingling in her anterior thigh. Plain dynamic radiography performed 6 months after the operation showed well-deposited PMMA without recollapse of the augmented vertebral body or any instability (Fig. 2B).

\section{DISCUSSION}

Avascular osteonecrosis is associated with infection, malignancy, radiation therapy, alcohol abuse, and use of systemic corticosteroid $^{1,5,11)}$. The present case did not have any of the predisposing factors associated with avascular osteonecrosis with the exception of osteoporosis. Avascular osteonecrosis associated with an osteoporotic VCF presents as an intravertebral vacuum cleft or collection of fluid on plain radiography or MR image $e^{2,5,8)}$. The early stage of avascular osteonecrosis is characterized by fluid and inflammatory exudate components, 
followed by vacuum in the latter stage ${ }^{5}$. Biopsy of avascular osteonecrosis showed necrotic cancellous bone and hyaline cartilage with fracture callus. Fibrin precipitation and fibrosis, although nonspecific, are also histologic features of avascular osteonecrosis ${ }^{5)}$.

Intravertebral fluid collection is considered as a manifestation of fluid transudation provoked by the inflammatory metabolites of damaged or dead cells after avascular osteonecrosis ${ }^{14)}$. Intravertebral fluid associated with avascular osteonecrosis presented as a well-defined hyperintense signal lesion on T2weighted sagittal MR image ${ }^{5,15)}$. Intravertebral fluid associated with osteoporotic avascular osteonecrosis mostly causes severe back pain due to intravertebral instability ${ }^{5,8)}$. Jang et al. ${ }^{5)}$ measured the changes in anterior vertebral height in patients with intravertebral pseudoarthrosis associated with osteoporotic avascular necrosis. All patients showed intravertebral instability on flexion-extension dynamic radiographs with a mean value of $5.9 \mathrm{~mm}$ (range, $2-11 \mathrm{~mm}$ ) and $87.5 \%$ of the total patients showed the presence of intravertebral fluid. Kim and $\mathrm{Kim}^{8)}$ reported a case of mobile vertebral fluid collection. Thirteen cc of fluid accumulated in the intravertebral cleft caused significant intravertebral instability, which was considered clinically malignant ${ }^{8,15}$. In the present case, dynamic radiographs could not be obtained due to intractable pain. Given that her back pain was aggravated while turning over and it was markedly relieved in the supine position, the presence of intravertebral instability associated with intravertebral fluid was highly suggested. The pathomechanism of epidural fluid collection is also strongly associated with dynamic instability due to intravertebral fluid. Dynamic instability of the vertebral body during flexion/extension may intermittently push the intravertebral fluid into the ventral epidural space through a fracture gap in the posterior cortex of the L1 vertebral body. The fracture gap in the posterior vertebral body might act like a one-way check valve and may contribute to the development of highpressure epidural fluid collection causing compression of the caudal equina, which provoked intractable pain in the buttock and the anterior thigh. MR myelography revealed the relationship among intravertebral fluid, epidural fluid collection, and the fracture gap in the posterior vertebral body which acted as a gate between the two fluids.

In the present case, the operation was performed in two steps; firstly, removal of the epidural fluid collection to decompress the cauda equine, and secondly, percutaneous vertebroplasty with PMMA to stabilize the intravertebral cleft and to prevent recurrence of the epidural fluid collection. Removal of the epidural fluid collection was not difficult since there was no capsule-like structure or a pseudo-membrane which usually adheres to the dural sac. Only on careful and gentle retraction of the dural sac, the epidural fluid suddenly gushed out from the ventral epidural space. Then, we performed vertebroplasty with injection of 3 cc of PMMA. Some surgeons try to aspirate the intravertebral fluid before PMMA injection ${ }^{5,8}$.
Since the injected PMMA in the intravertebral fluid may form a solid lump rather than a contiguous bone interdigitation, injection of PMMA without aspiration of intravertebral fluid may aggravate the process of osteonecrosis and result in recollapse of the augmented vertebra ${ }^{4)}$. When the intravertebral fluid is less, vertebroplasty can be successfully performed without aspiration of the intravertebral fluid" ${ }^{5)}$. We did not try to aspirate the intravertebral fluid before injection of PMMA into the intravertebral cleft. MR image performed on the day after the operation showed that the intravertebral fluid had nearly disappeared and PMMA had filled the cleft instead. Injected PMMA was placed into the intravertebral cleft and it pushed the intravertebral fluid into the epidural space through the fracture gap in the posterior cortex.

Short-term results of percutaneous vertebroplasty for avascular osteonecrosis associated with an osteoporotic VCF were reported to show high effectiveness ${ }^{5,6,9-11)}$. The study by Jang et al.", with a mean follow-up duration of 11 months, showed that percutaneous vertebroplasty was a minimally invasive and effective procedure that provides pain relief and stabilization of spinal instability associated with osteoporotic avascular osteonecrosis. In contrast, some surgeons recommend instrumented spinal fusion since long-term results of percutaneous vertebroplasty were disappointing ${ }^{3,4,7,12,13)}$. Heo et al. ${ }^{4}$ analyzed at least 2-year follow-up results of percutaneous vertebroplasty for avascular osteonecrosis with an osteoporotic VCF. Although vertebroplasty appears to be a good treatment during the early follow-up period (within 1 year), it may not provide sufficient stability for 2 years or longer. Given the high incidence of perioperative medical complications and instrument failure due to severe osteoporosis, instrumented fusion surgery is a challenge in elderly patients with an osteoporotic VCF ${ }^{12)}$. Nakajima et al. ${ }^{12)}$ reported postoperative complications in approximately $25 \%$ of patients, and they were mainly related to instrumentation. To overcome the complications associated with extensive spinal fusion, Kim and $\mathrm{Heo}^{7}$ performed short-segment pedicle screw fixation with PMMA augmentation after postural reduction. Surgical results of short-segment fixation were promising. Vertebral height and kyphotic angle of the compressed vertebrae were significantly corrected, and restored vertebral height was maintained for more than 2 years. The postoperative compression ratio and kyphotic angle were significantly lower compared with those in the percutaneous vertebroplasty group ${ }^{7}$. However, instrument-related complications were observed in 2 of the 18 cases (11.1\%). Given the old age, poor bone quality, and high chance of possible medical complications, percutaneous vertebroplasty was a more reasonable and safe choice in the present case. Six months after the procedure, the patient's condition was still good and plain radiography did not show recollapse of the PMMA-augmented vertebra. Nonetheless, careful observation of the patient with further long-term follow-up is necessary. 


\section{CONCLUSION}

Epidural fluid collection which originated from avascular osteonecrosis associated with an osteoporotic VCF caused intractable pain in the buttock and the anterior thigh. Posterior hemilaminectomy and drainage of the epidural fluid followed by percutaneous vertebroplasty was an effective treatment.

\section{CONFLICTS OF INTEREST}

No potential conflict of interest relevant to this article was reported.

\section{REFERENCES}

1. Chou LH, Knight RQ: Idiopathic avascular necrosis of a vertebral body. Case report and literature review. Spine (Phila Pa 1976) 22:1928-1932, 1997

2. Dupuy DE, Palmer WE, Rosenthal DI: Vertebral fluid collection associated with vertebral collapse. AJR Am J Roentgenol 167: 1535-1538, 1996

3. Heo DH, Chin DK, Yoon YS, Kuh SU: Recollapse of previous vertebral compression fracture after percutaneous vertebroplasty. Osteoporos Int 20:473-480, 2009

4. Heo DH, Choi JH, Kim MK, Choi HC, Jeong JH, Chin DK, et al.: Therapeutic efficacy of vertebroplasty in osteoporotic vertebral compression fractures with avascular osteonecrosis: a minimum 2-year follow-up study. Spine (Phila Pa 1976) 37:E423E429, 2012

5. Jang JS, Kim DY, Lee SH: Efficacy of percutaneous vertebroplasty in the treatment of intravertebral pseudarthrosis associated with noninfected avascular necrosis of the vertebral body. Spine (Phila Pa 1976) 28:1588-1592, 2003

6. Kim DY, Lee SH, Jang JS, Chung SK, Lee HY: Intravertebral vacuum phenomenon in osteoporotic compression fracture: report of 67 cases with quantitative evaluation of intravertebral instability. J Neurosurg 100:24-31, 2004

7. Kim HS, Heo DH: Percutaneous pedicle screw fixation with polymethylmethacrylate augmentation for the treatment of thoracolumbar intravertebral pseudoarthrosis associated with Kummell's osteonecrosis. Biomed Res Int 2016:3878063, 2016

8. Kim SW, Kim HS: Mobile vertebral fluid collection associated with compression fracture. Arthritis Rheum 65:1949, 2013

9. Lane JI, Maus TP, Wald JT, Thielen KR, Bobra S, Luetmer $\mathrm{PH}$ : Intravertebral clefts opacified during vertebroplasty: pathogenesis, technical implications, and prognostic significance. AJNR Am J Neuroradiol 23:1642-1646, 2002

10. Libicher M, Appelt A, Berger I, Baier M, Meeder PJ, Grafe I, et al.: The intravertebral vacuum phenomen as specific sign of osteonecrosis in vertebral compression fractures: results from a radiological and histological study. Eur Radiol 17:2248-2252, 2007

11. Lieberman IH, Dudeney S, Reinhardt MK, Bell G: Initial outcome and efficacy of "kyphoplasty" in the treatment of painful osteoporotic vertebral compression fractures. Spine (Phila Pa 1976) 26:1631-1638, 2001

12. Nakajima H, Uchida K, Honjoh K, Sakamoto T, Kitade M, Baba $\mathrm{H}$ : Surgical treatment of low lumbar osteoporotic vertebral collapse: a single-institution experience. J Neurosurg Spine 24:3947, 2016

13. Park SJ, Kim HS, Lee SK, Kim SW: Bone cement-augmented percutaneous short segment fixation: An effective treatment for Kummell's disease? J Korean Neurosurg Soc 58:54-59, 2015

14. Sweet DE, Madewell JE. Osteonecrosis: Pathogenesis. In: Resnick D ed. Diagnosis of bone and joint disorders, ed 3rd. Philadelphia, PA: W.B. Saunders Company, 1995, pp3445-3494

15. Thawait SK, Marcus MA, Morrison WB, Klufas RA, Eng J, Carrino JA: Research synthesis: what is the diagnostic performance of magnetic resonance imaging to discriminate benign from malignant vertebral compression fractures? Systematic review and meta-analysis. Spine (Phila Pa 1976) 37:E736-E744, 2012 